\title{
Additive Manufacturing Enabled by Electrospinning for Tougher Bio-Inspired Materials
}

\author{
Komal Agarwal, ${ }^{1}$ Yinning Zhou, ${ }^{2}$ Hashina Parveen Anwar Ali, ${ }^{2}$ Ihor Radchenko, ${ }^{2}$ \\ Avinash Baji $\mathbb{D}^{1,3}$ and Arief S. Budiman $\mathbb{D}^{2}$ \\ ${ }^{1}$ Engineering Product Development Pillar, Singapore University of Technology and Design, Singapore 487372 \\ ${ }^{2}$ Xtreme Materials Laboratory (XML), Singapore University of Technology and Design, Singapore 487372 \\ ${ }^{3}$ Department of Engineering, School of Engineering and Mathematical Sciences (SEMS), La Trobe University, \\ Bundoora 3086, Australia \\ Correspondence should be addressed to Avinash Baji; a.baji@latrobe.edu.au and Arief S. Budiman; suriadi@alumni.stanford.edu
}

Received 13 June 2018; Accepted 24 September 2018; Published 1 November 2018

Academic Editor: Jose M. Cabrera

Copyright (C) 2018 Komal Agarwal et al. This is an open access article distributed under the Creative Commons Attribution License, which permits unrestricted use, distribution, and reproduction in any medium, provided the original work is properly cited.

\begin{abstract}
Nature has taught us fascinating strategies to design materials such that they exhibit superior and novel properties. Shells of mantis club have protein fibres arranged in a 3D helicoidal architecture that give them remarkable strength and toughness, enabling them to absorb high-impact energy. This complex architecture is now possible to replicate with the recent advances in additive manufacturing. In this paper, we used melt electrospinning to fabricate $3 \mathrm{D}$ polycaprolactone (PCL) fibrous design to mimic the natural helicoidal structures found in the shells of the mantis shrimp's dactyl club. To improve the tensile deformation behavior of the structures, the surface of each layer of the samples were treated with carboxyl and amino groups. The toughness of the surfacetreated helicoidal sample was found to be two times higher than the surface-treated unidirectional sample and five times higher than the helicoidal sample without surface treatment. Free amino groups $\left(\mathrm{NH}_{2}\right)$ were introduced on the surface of the fibres and membrane via surface treatment to increase the interaction and adhesion among the different layers of membranes. We believe that this represents a preliminary feasibility in our attempt to mimic the 3D helicoidal architectures at small scales, and we still have room to improve further using even smaller fibre sizes of the modeled architectures. These lightweight synthetic analogue materials enabled by electrospinning as an additive manufacturing methodology would potentially display superior structural properties and functionalities such as high strength and extreme toughness.
\end{abstract}

\section{Introduction}

Natural biological composites have captured tremendous scientific interest in recent years among researchers to develop high-performance synthetic composites [1-4]. Mimicking the structure and design of the natural composites that are found in connective tissues, bones, and exoskeletons can offer fascinating prospects for designing strong and novel synthetic composites $[4,5]$. The natural composites are obtained by the self-assembly process and are made of macromolecular building blocks. At the structural levels, the molecules are self-assembled to obtain a higher order micro- or macrostructure [4].

In a recent study, Weaver et al. attributed the high toughness and strength of mantis shrimp's dactyl club to its microstructure and unique arrangement of protein fibres within the structures. Investigations have also attributed the mechanical strength and toughness of the material to the complex interplay between structure, stiffness, strength, and impact mechanics of the dactyl club [6]. Their findings show that the club is divided into three distinct regions viz. outer crystalline apatite region, chitin/amorphous calcium carbonate (ACC) periodic region in the form of rotated plywood, and mineralized chitin fibrils striated region. The periodic mineralized chitin fibril region that shows a helicoidal architecture (Figure 1(b)) can dissipate energy from the impact region through quasi-plastic compressive responses. Therefore, it provides a fracture-toughening barrier that hinders the catastrophic propagation of microcracks when subjected to repeated impacts [6,7]. Wilts et al. in their article demonstrated that such helicoidal architecture is 


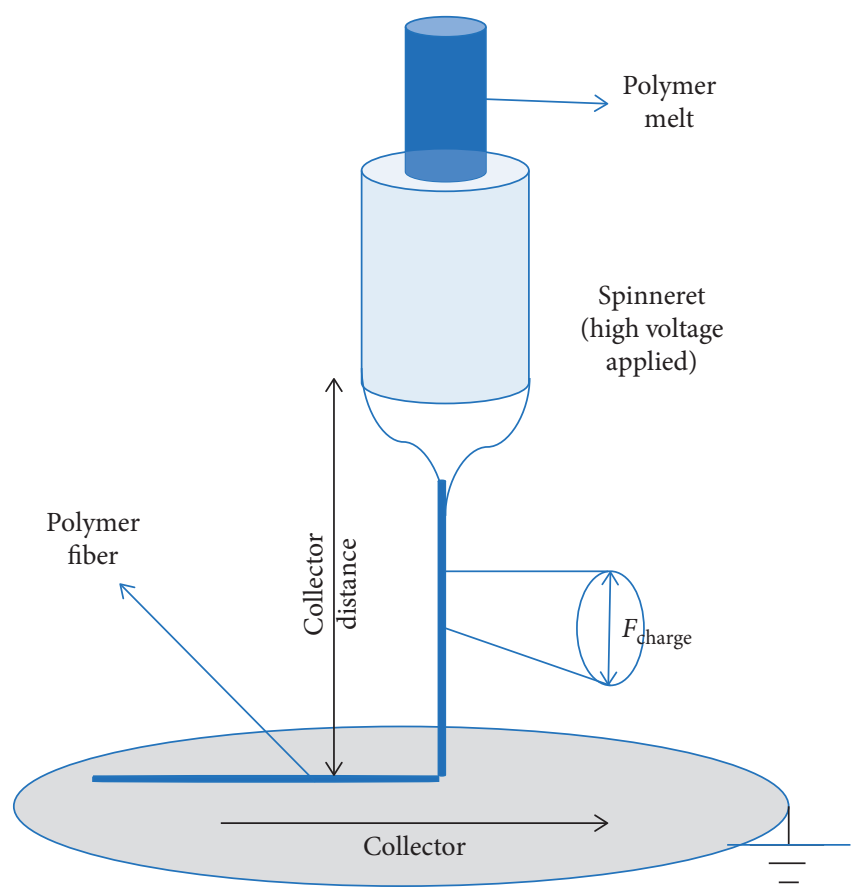

(a)



(b)

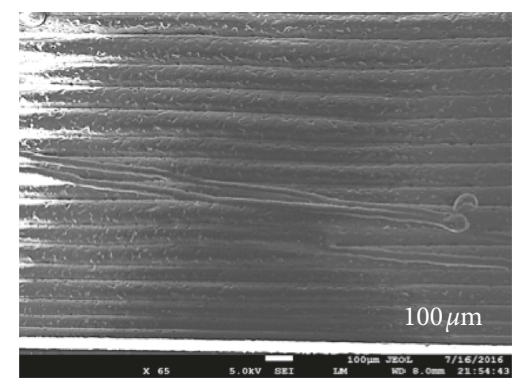

(c)

FIGURE 1: Methodology, design, and SEM images of one layer of 3D Helicoidal Structure. (a) Schematics of near-field melt electrospinning (NFES) - the process used to first lay down patterns of electrospun fibres on top a collector; (b) illustration of a top view of the hierarchical helicoidal design of the basic building blocks found in shells of mantis shrimp dactyl club, as reported in the literature; (c) scanning electron microscope (SEM) image of a layer of highly aligned, dense fibre-structured samples of melt electrospinning fibres with diameters around $10 \mu \mathrm{m}$ at $65 \mathrm{x}$ magnification.

commonly found in some plants and animal species [8]. They not only attribute the optimal properties and structural color to this helicoidal arrangement but also attribute the impact toughness to such structures. The studies indicate that the remarkable strength (rigidity) and toughness of the exoskeletons is attributed to the hierarchical design of the basic building blocks as illustrated in Figure 1(b). The fibres are assembled into bundles to form horizontal planes, which are then superimposed to obtain helicoidal stacking [9-11].

Mimicking this structured design could lead to the development of synthetic impact resistant materials. Although some studies have characterized the mechanical deformation behavior of the natural material, very few studies have concentrated their efforts on fabricating synthetic structures $[6,7,12]$. Gu et al. [13] created nacre-like polymer composite structures using $3 \mathrm{D}$ printing where layers were stacked with orientation angles of 0 and 90 degrees to generate a laminate construct. These nacre-like designs outperform the constituent materials in impact resistance. Yaraghi et al. [14] used 3D printing to mimic the natural 3D helicoidal architecture. However, the sizes of fibres in this study are in the millimeter size domain. In this study, we use near-field solution electrospinning as shown in Figure 1(a) to fabricate the synthetic 3D hierarchical structures by precisely depositing the fibres in a controlled fashion. By controlling the processing variable, the size of the fibres can be in smaller scales (hundreds to tens of microns). This approach of using electrospinning as an additive manufacturing methodology offers the most versatile and promising technique that enables the fabrication of the hierarchical fibres even to the nanometer regimes. From the literature, both fibre structures $[15,16]$ as well as the smaller scaled microstructures [17-20] of material have been known to lead to unprecedented materials properties, including toughness and other damage-resistant behavior [21-26].

We believe that the helicoidal structure adapts to tensile stress by allowing the plane of fibres to rotate toward or away from the applied tensile load, prolonging the final catastrophic events of failures. The reason behind the unique strength and toughness of the structure is its ability to adapt to the loading stress. Thus, we establish a fundamental understanding of the fracture-delaying mechanisms in the 3D hierarchy microarchitectured advanced materials enabled by additive manufacturing to achieve remarkable structural properties and functionalities.

Strengthening the biomimetic 3D hierarchical nano- and microscale architectures, enabled by additive manufacturing, is the unique aspect of this article. The development of such lowcost, lightweight, high-performance materials will be highly useful for protective technologies such as in soldier body armors, sports/athletic gears, and aerospace/aircraft applications.

\section{Experimental}

2.1. Near-Field Melt Electrospinning (NFES). A custom-built near-field melt electrospinning setup was used to fabricate 
the samples. Briefly, polycaprolactone (PCL, molecular weight $=45 \mathrm{kDa}$, Sigma-Aldrich, Singapore) pellets were fed into a metallic syringe surrounded by a hot water jacket system (NanoNC, Korea). The temperature was raised to $80^{\circ} \mathrm{C}$ to melt the polymer. Following this step, a high voltage power supply was connected to the needle. The flow rate and the voltage used during the electrospinning were $20 \mu \mathrm{l} / \mathrm{h}$ and $9 \mathrm{kV}$, respectively. A syringe pump (EQ-500SP-H Syringe Pump, Premier Solution Pte Ltd.) was used to dispense the PCL from the metallic syringe. The distance between the tip of the needle and collector was $2 \mathrm{~cm}$. The fibres were collected on an aluminum plate placed on a XY linear computerintegrated motorized stage (Zugo Photonics, Singapore). The speed of motorized stage was set at $100 \mathrm{~mm} / \mathrm{s}$ at $30 \mu \mathrm{m}$ microstep intervals. All the experiments were conducted at room temperature $\left(17-22^{\circ} \mathrm{C}\right)$ within a closed chamber. The experimental conditions were kept constant for all the experiments. A vertical electrospinning setup was adopted (having only one axis to oscillate) to obtain highly uniform and dense fibres as shown in Figure 1(c). Seven layers of such aligned array of fibres were collected and manually stacked on top of each other to mimic the natural helicoidal structure. Each layer was placed such that the longitudinal direction of the fibres was rotated by $15^{\circ}$ [24] with respect to the longitudinal direction of the fibre just below it. Following this, the sample was hot-pressed at $45^{\circ} \mathrm{C}$ and $0.2 \mathrm{MPa}$ pressure using a manual hydraulic press with digital heating plates (Model 4386, Carver Inc. USA) to obtain the helicoidal structures as shown in Figure 2. A control sample was also prepared by stacking the seven layers of fibre arrays on top of each other followed by hot-pressing. In this set of samples, the fibres in each layer were unidirectionally oriented.

2.2. Surface Treatment. Another set of samples were prepared by functionalizing the surface of the fibres before they were hotpressed. Briefly, once the aligned arrays of fibres were obtained, carboxyl groups were introduced on the surface of the fibres by treating the fibres with low-pressure air plasma for 5 minutes using the electrodeless, inductively coupled RFGD instrument (PDC-002; Harrick Plasma, Ithaca, NY, USA) [27]. Following this, amino groups were introduced by immersing the samples in $10 \%(\mathrm{w} / \mathrm{v})$ solution of 1,6-hexanediamine prepared in isopropanol at $37^{\circ} \mathrm{C}$ for $1 \mathrm{~h}$ [28]. Distilled water was used to wash the samples. The samples were then dried under vacuum at room temperature for two hours before they were hot-pressed to obtain helicoidal samples. A control sample was prepared by stacking the surface-treated fibre array on top of each other followed by hot-pressing. The fibres in each layer were unidirectionally oriented.

2.3. Characterization. The cross section of the helicoidal samples is investigated by fracturing the samples under liquid nitrogen and imaging them using a scanning electron microscope (SEM). To view the microstructure of the samples, the samples were sputter-coated with gold $(18 \mathrm{~mA}$, 90 seconds) before their structures were examined using a scanning electron microscope (Tescan, accelerating voltage of $10 \mathrm{kV}$ ).
The tensile deformation behavior of the samples was investigated using a Zwick Roell (Zwick Roell, Ulm, Germany) Z0.5 static testing machine (Figure 3(a)). All four samples, viz. helicoidal PCL fibre samples with and without surface treatment and unidirectionally oriented PCL samples with and without surface treatment with identical dimensions $\left(4 \times 2.5 \times 0.6 \mathrm{~mm}^{3}\right.$ width, length, and thickness) were tested. For comparison purposes, a bulk PCL sample was also prepared by hot-pressing the PCL pellets and its deformation behavior was compared with the other samples. The sample ends were glued to acrylic slabs prior to the tests as shown in Figures 3(c) and 3(d). This setup was used to ensure that the samples were firmly gripped during the tension tests. The tensile test was performed at room temperature under displacement control at a strain rate of $=$ $100 \mu \mathrm{m} / \mathrm{min}$ and a preload of $0.1 \mathrm{~N}$.

A differential scanning calorimeter (DSC) from TA Instruments, DSC Q100, was used to determine the crystallinity and melting behavior of the PCL samples. The temperature was ramped at $3^{\circ} \mathrm{C} / \mathrm{min}$ from $0^{\circ} \mathrm{C}$ temperature to $80^{\circ} \mathrm{C}$ under a nitrogen atmosphere. The thermal degradation temperature of the helicoidal PCL sample and bulk PCL was determined using thermogravimetric analyzer (TGA, TA Q50). The weight loss of the samples as a function of temperature was recorded at a heating rate of $10^{\circ} \mathrm{C} / \mathrm{min}$ in the $\mathrm{N}_{2}$ atmosphere.

\section{Results and Discussion}

Figure 2 clearly illustrates the cross section of partial 3D helicoidal structures with $15^{\circ}$ rotations between each layer. The red dashed lines shown in the SEM image illustrate the different layers within the sample and demonstrate that the sample is composed of multiple layers of fibre arrays. It is evident that the cross section of the fibres in each region of the sample shows variation from circle to ellipse and then becomes a column.

Our objective is to investigate the mechanisms leading to the enhanced mechanical properties of the 3D hierarchical microarchitectured materials inspired by nature while maintaining other desirable properties and/or functionalities.

The tensile tester is used to investigate the tensile deformation behavior of the samples. Digital images of the samples are taken during the deformation process. Figure 4 shows various representative stages of the tensile test in (a) bulk PCL, (b) unidirectionally oriented PCL fibre sample, and (c) helicoidal PCL fibre sample. Figure 5 shows the representation of stress vs. strain curves recorded for the samples. Each of the tensile stress-strain curves shown in Figure 5 is the representative of a few samples (between 5 and 8 samples) in each of the groups specified (bulk, unidirectionally oriented, helicoidal, unidirectionally oriented with surface treatment, and helicoidal with surface treatment). Within each group, the stress-strain curves showed self-consistent characteristics as well as similar ranges of magnitudes of the strengths and ductility (with differences between the samples within the group not more than $\pm 6 \%$ for the max stress values, as shown with the experimental error bars in Figure 5). For clarity and simplicity, Figure 5 shows only the representatives of each of the groups (but their variations are indicated by the 


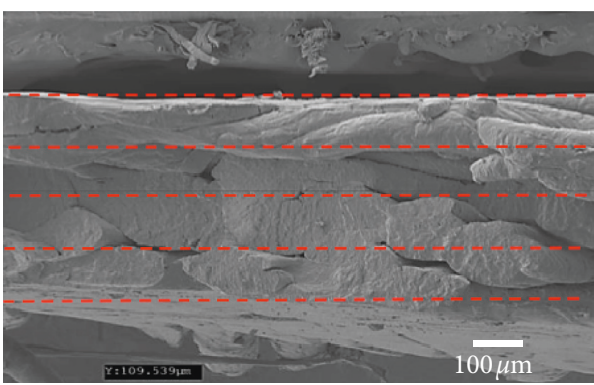

(a)

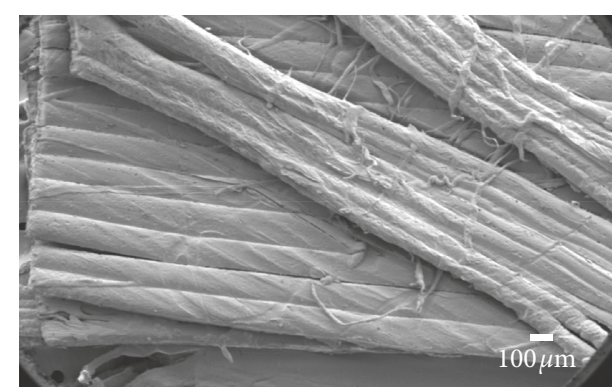

(b)

FIGURE 2: SEM image of the 3D helicoidal structure with $15^{\circ}$ rotations between each layer. (a) SEM image showing cross section of the samples with a few stacked layers of fibre aligned at different direction at 95x magnification (the red-dashed lines are to indicate the boundary between layers), demonstrating of the basic feasibility to synthesize the full 3D helicoidal fibre structures similar to the natural material; (b) SEM image of the sample viewed from the top.

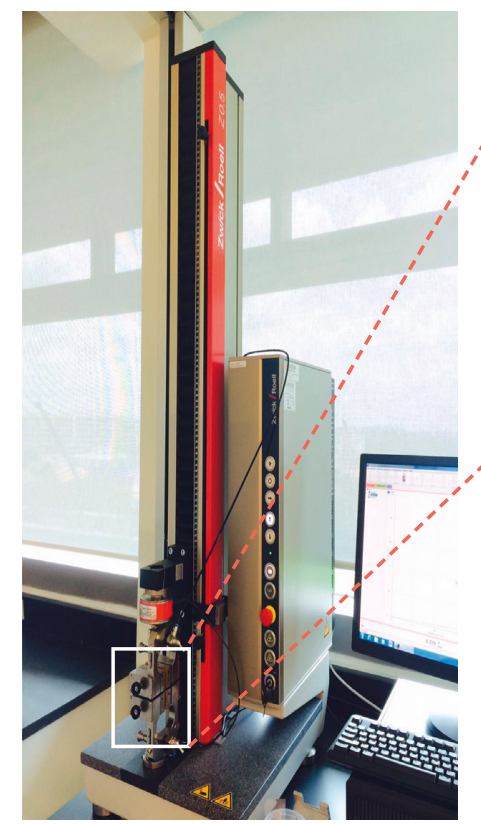

(a)



(b)

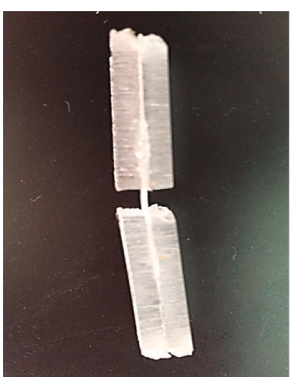

(c)



(d)

Figure 3: Tensile test setup and sample preparation. (a) Zwick Roell static testing machine for tensile test. (b) Partial magnification of sample placing. (c) Side view of tensile test sample, four pieces of acrylic slabs with super glue were used to attach the samples. (d) Top view of tensile test sample.

experimental error bars shown at max stress values and subsequently at strain values of $200 \%, 400 \%$, and $600 \%)$. They show unique characteristics, which are distinctive enough from group to group such as evident and represented in Figure 5. The toughness of the samples is determined by measuring the area under the stress-strain curves of the samples. It is clear that the bulk sample demonstrates elastic deformation as well as plastic deformation. For the bulk PCL, a crack initiated at the edge of the sample and propagated perpendicular to the applied tensile stress. For the sample with unidirectionally oriented fibre arrays, all the fibres arrays are aligned along with the tensile loading direction. Thus, this sample demonstrated higher yield strength as the load is shared equally by all the fibre arrays, as shown in Figure 5(a). The digital images show that not all the fibres fractured at the same time. This could be because of some misalignment.
The stress vs. strain curve of the helicoidal PCL fibre sample (without surface treatment) shows that the yield stress is lower than the bulk sample, as shown in Figure 5(a). We believe that this can be attributed to the poor adhesion between the fibres. Unlike the bulk PCL and the unidirectional PCL fibre samples, the helicoidal PCL fibre samples have significantly lower adhesion between the layers due to the different orientation of the fibres in each of the layers. To investigate this hypothesis, the surface of the fibres is functionalized and then their tensile deformation behavior is investigated as described in the subsequent paragraphs as well as shown in Figure 5(b).

Although the helicoidal PCL fibre samples showed lower yield strength, it is evident from Figure 5(a) that these samples exhibit extended ductility compared to the other two control samples. Furthermore, the digital images taken 


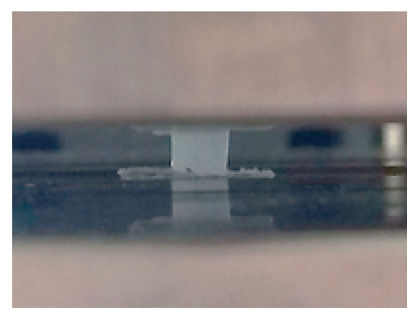

(a1)

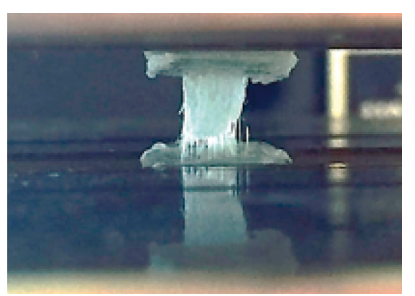

(b1)

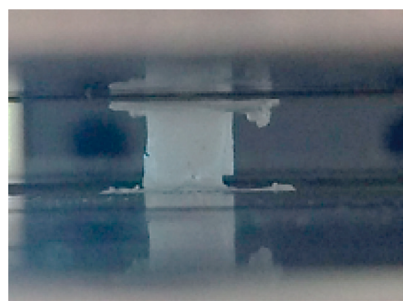

(c1)

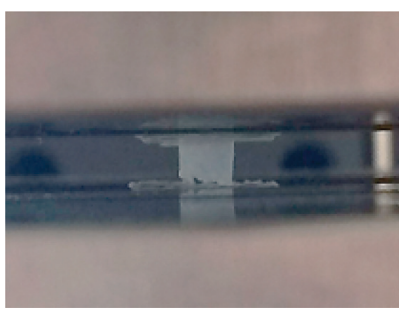

(a2)



(b2)

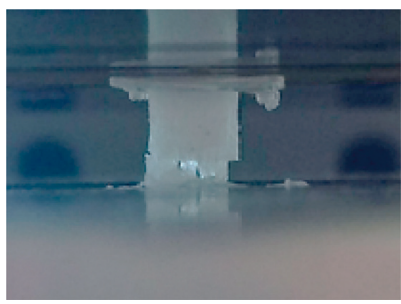

(c2)



(a3)

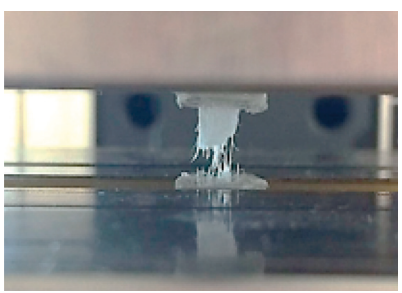

(b3)

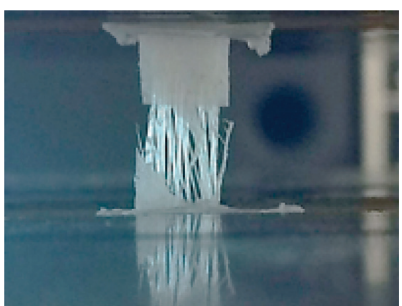

(c3)



(a4)

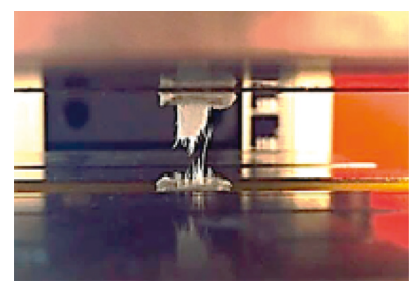

(b4)



(c4)

FIGURE 4: Various stages of tensile deformation of polycaprolactone (PCL) samples. (a1-a4) Bulk PCL. (b1-b4) Unidirectionally oriented PCL sample, composed of fibres aligned along the loading axis. (c1-c4) Helicoidal PCL sample, composed of fibres aligned at different angles forming helicoidal structure.



(a)

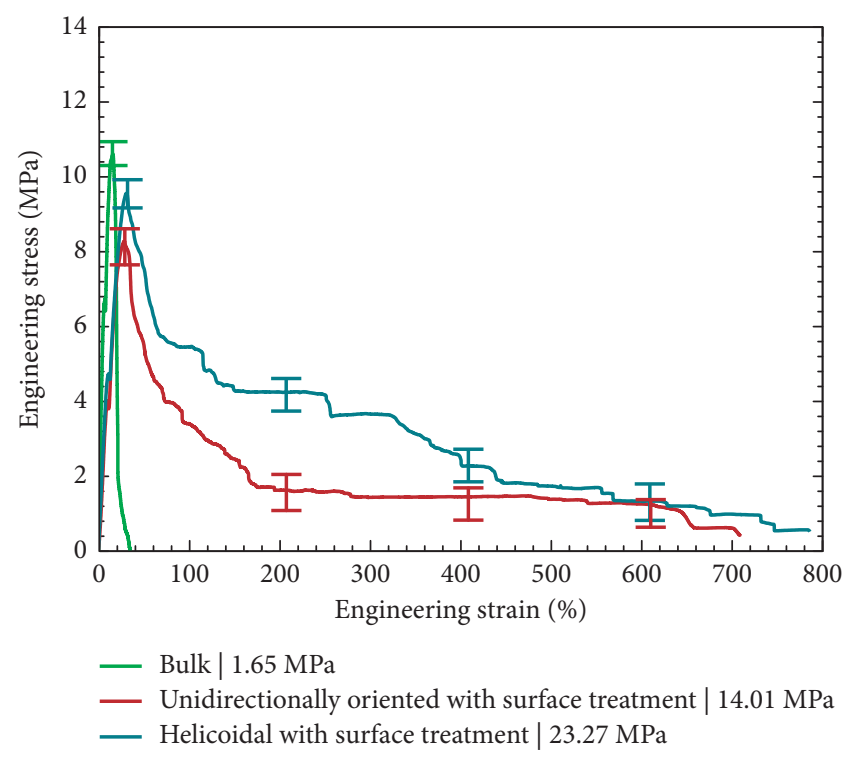

(b)

Figure 5: Stress-strain curves and toughness measurements. (a) Bulk PCL in comparison with unidirectionally oriented PCL fibre sample and helicoidal PCL fibre sample without surface treatment. (b) Bulk PCL in comparison with surface-treated unidirectionally oriented PCL fibre sample and surface-treated helicoidal PCL fibre sample. The experimental error bars are provided here to indicate variations within each group of the samples at max stress values and subsequently at strain values of $200 \%, 400 \%$, and $600 \%$. The variations of the max stress values in each of the groups are not more than $\pm 6 \%$. The toughness, as defined by the area under the stress-strain curve, for each of the groups of samples has been provided in the legend (the toughness is presented here with the unit of MPa $\times$ unitless strain). 
during the deformation process show that the fibres within the different layers of the materials were reoriented along the loading direction in different stages during deformation. This explains why the fracture of the sample was delayed compared to the bulk sample. The fracture of the helicoidal PCL fibre samples took 5 times longer than the unidirectionally orientated samples and nearly 10 times longer than the bulk PCL samples. The final failure of the helicoidal PCL samples is preceded by twisting of the fibres and dramatical elongation of the fibres before fracture.

The areas under the stress vs. strain curve (i.e., the toughness) of the bulk PCL, unidirectionally oriented PCL, and the PCL helicoidal structures are measured to be 1.65 , 5.27 , and $8.07 \mathrm{MPa}$ (the area under the stress-strain curve is presented here with the unit of MPa x unitless strain), respectively. Thus, the toughness of the PCL helicoidal structures is at least 5 times and 1.5 times higher than those of the bulk PCL and the unidirectionally oriented PCL samples, respectively. The helicoidal PCL fibre sample achieved nearly $650 \%$ strain deformation while unidirectionally oriented PCL fibre sample reached $100 \%$ strain deformation and bulk PCL only appeared to achieve about $30 \%$ strain deformation, as shown in Figure 5(a).

The effect of the surface treatment as alluded earlier in this manuscript is shown in Figure 5(b). The surface-treated unidirectionally oriented PCL samples showed increased tensile strength and extended ductility compared to their nonsurface-treated counterparts, whereas the surface-treated PCL helicoidal samples showed comparable yield strength to the bulk PCL as well as surface-treated unidirectional PCL samples, as evident in Figure 5(b), while maintaining similar benefits in the tensile deformation behavior (i.e., delayed fracture events) with even some further improvements in extended ductility compared to their non-surface-treated counterparts. The surface-treated unidirectional PCL samples achieved extended ductility of nearly $800 \%$ as shown in Figure 5(b). The toughness value of the surface-treated unidirectionally oriented PCL sample and surface-treated PCL helicoidal sample is determined to be 14.01 and $23.27 \mathrm{MPa}$.

These results show that the surface treatment of the fibres improved the overall toughness of the sample during tensile testing. The toughness of surface-treated unidirectionally oriented PCL sample increased by 2.6 times compared to the unidirectionally oriented PCL sample. The surface-treated helicoidal PCL sample showed an increase in toughness by almost 3 times compared to the 3D helicoidal PCL sample without surface treatment and by almost 1.6 times higher than surface-treated unidirectionally oriented PCL samples, accompanied by a substantial increment of engineering stress.

Although the surface treatment seems to result in improvements in both the unidirectionally oriented PCL samples and helicoidal PCL samples (as represented by the red and blue curves in Figure 5(b), respectively), we observed a unique characteristic of the blue curves. This characteristic is signified by the broad "shoulder" that only gradually got lower and lower as each of the layers (with the different fibre orientations) was gradually extended to the maximum possible. In contrast, the red curves could be characterized by a much narrower "shoulder" that quickly gets down and stays down until the last strain of fibres was broken. Although the total extended ductility of the two representative curves was similar as shown in Figure 5(b), the areas under the curves of the blue curves are always about $40-50 \%$ higher, which indicates the significant difference between these two groups of samples.

This unique characteristic is also evident from the tortuosity of the breaking paths as shown in images of Figure 4(c) of the helicoidal PCL samples especially. To substantially reduced extent, the unidirectional PCL samples exhibit similar behaviors, but as the evidence from the calculations of the areas under the curves, the toughness of the blue curves is always about $40-50 \%$ higher than those of the red curves. In addition, Figure $5(\mathrm{a})$ provides further evidence that the helicoidal architecture indeed uniquely leads to the higher performance. Even without surface treatment, the few samples in the "Helicoidal" group in Figure 5(a) have already consistently shown the characteristics of "broad shoulders" and gradual reduction of strength with extended strains, such as illustrated above with the blue curve in Figure 5(b). The surface treatment just elevates the overall stress-strain curves into a higher level of strengths of the materials. We, therefore, think all these evidences are strong indications that the helicoidal fibre orientation matters in addition to the surface treatment effects.

The stress-strain curves here thus showed promising results of the feasibility of the helicoidal PCL samples over both bulk and unidirectionally oriented PCL samples. As the PCL sample is being pulled in tension, a crack is initiated and propagated in the direction perpendicular to the loading axis. In theory, fibres in the transverse direction are least likely to break over longitudinal direction along the loading. For the unidirectionally oriented sample, since all the fibres were in the same direction as the loading axis, it was easy for the crack to be propagated through the fibres. The individual fibres were easy to rip due to the same orientation. Due to the rotated fibre alignment directions in the layers in the helicoidal samples, it is more difficult for the crack to propagate and further proceed to the final catastrophic events. As the layers of fibres keep changing directions, crack propagation from one layer to the next is effectively delayed as it keeps losing its primary driving force. Each layer of fibres is essentially propagating the crack only after further tensile straining effectively rotates its fibre alignment to become normal to the loading axis (i.e., the weakest configuration). But at the same time, as these layers rotate to weaker configurations, other layers rotate to stronger configurations (when the fibre alignment is parallel to the loading axis). These mechanisms are evident from the tortuosity of the breaking paths as shown in images of Figure 4(c) of the helicoidal PCL samples especially (although to some reduced extent, the unidirectional PCL samples also exhibit similar behaviors). These mechanisms basically represent an effective means of energy and thus damage dissipation, which could further lead to an effective mechanism for impact resistance.

Surface adhesion between the individual PCL fibres and layers plays a significant role in the mechanical performance of these biomimetic materials. This is evident from 


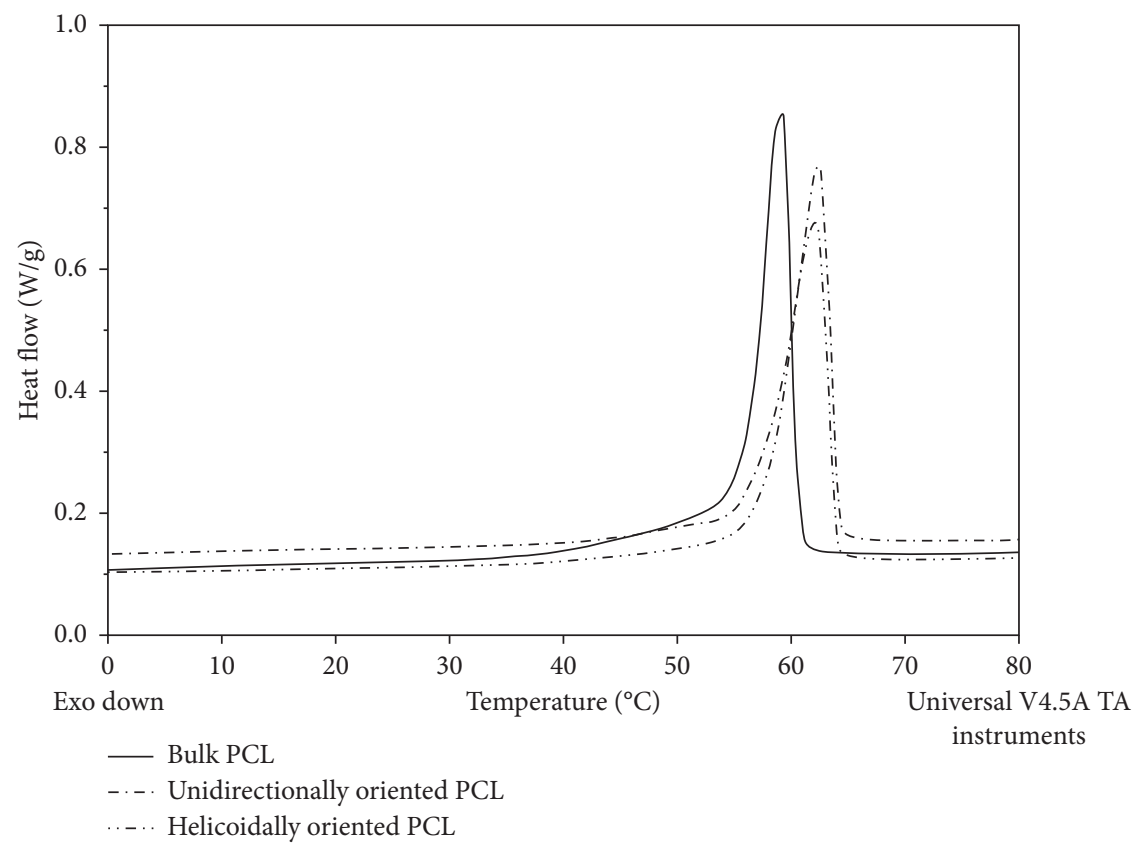

FIgURE 6: DSC scans of bulk, helicoidal, and unidirectionally oriented PCL samples.

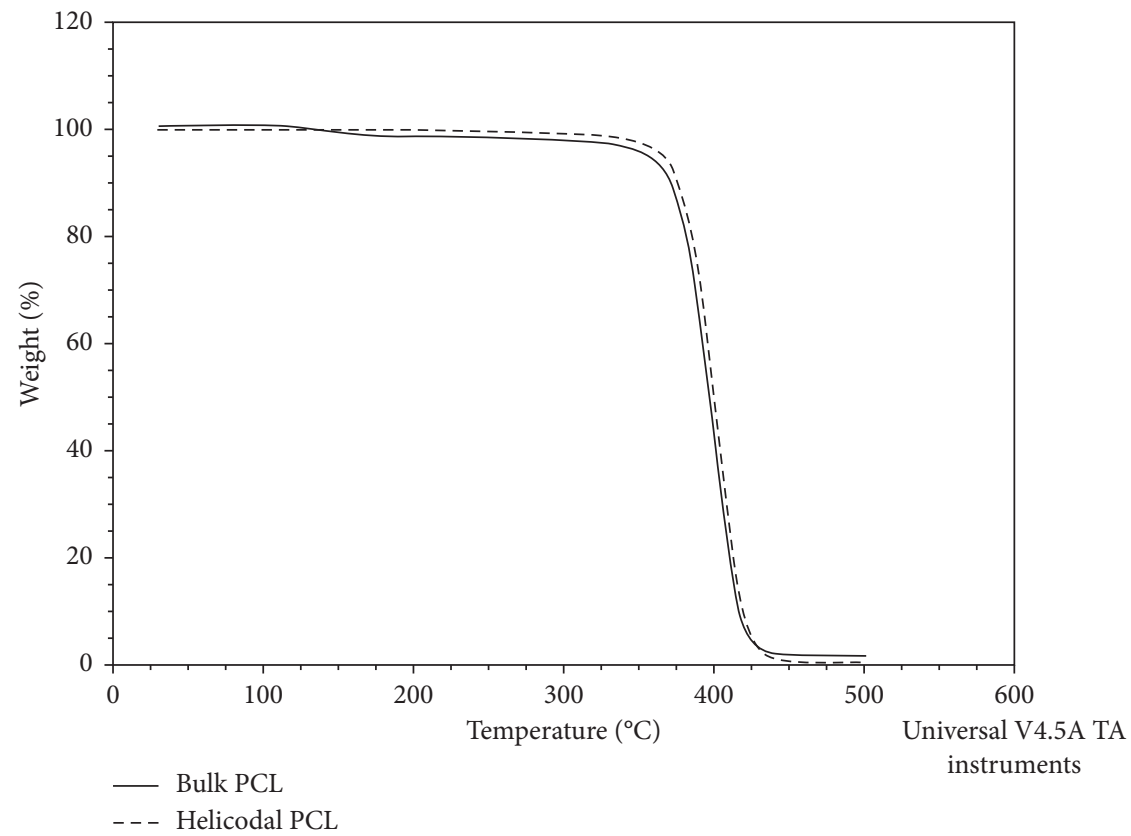

FIgURE 7: TGA curves of bulk and helicoidal PCL.

the fracture behavior of surface-treated versus the nonsurface-treated samples, as illustrated in Figures 5(a) and 5(b). After the fibre surface treatment, free amino groups were introduced on the PCL membrane [28]. The interaction between the oppositely charged carboxyl group and amino groups helped to improve the adhesion between the fibres and the individual layers. This shows the importance of further studies in the interplay between all the microstructural building blocks in mimicking the $3 \mathrm{D}$ helicoidal alignment of the exoskeleton structure of the mantis dactyl club in the design of enhanced fracture toughness of the biomimetic synthetic materials.

The thermal properties of the samples are also investigated and the effect of helicoidal architecture on the crystallinity, melting temperature, and thermal degradation is determined. Figure 6 shows the DSC thermal scans of the bulk PCL, unidirectionally oriented PCL, and helicoidal PCL samples. It is clear that the melting temperature of the helicoidal and unidirectionally oriented PCL is around $62^{\circ} \mathrm{C}$ while the melting temperature of bulk PCL is $59^{\circ} \mathrm{C}$. The 
lower melting temperature recorded for bulk PCL indicates the presence of smaller crystallite structure within the sample. It is also obvious that the peak in the case of bulk PCL is sharper than the peaks recorded for the other two samples. This indicates that bulk PCL has smaller average crystallite size [29]. Melting enthalpy of the samples is measured from the DSC curves to determine the crystallinity. Melting enthalpy of pure crystalline PCL is obtained from literature to be $136 \mathrm{~J} / \mathrm{g}$ [30]. The crystallinity of bulk PCL is determined to be $45.7 \%$, while the crystallinity of helicoidal PCL and unidirectionally oriented PCL is determined to be $39.7 \%$ and $42 \%$, respectively. This indicates that although the crystallite size in bulk PCL is smaller, the crystalline content is much higher than the other two samples. The lower crystallinity in helicoidal and unidirectionally oriented PCL can be attributed to the electrospinning process. During melt electrospinning, PCL is heated to a higher temperature and deposited on the collector at ambient temperature. This quenching of the material restrains the crystal formation and is responsible for the lower crystallinity displayed by the helicoidal and unidirectionally oriented PCL fibres. It is interesting to note that even though the helicoidal PCL has a lower percentage of crystallinity, it displays better tensile properties. This indicates that the helicoidal architecture plays a major in influencing the tensile properties of the helicoidal PCL. Figure 7 shows the TGA curves of helicoidal and bulk PCL. The curves show that the degradation behavior of both samples is identical and both samples degrade at identical temperatures.

\section{Conclusion}

In summary, we have proven the technical feasibility to create a synthetic analogue of 3D helicoidal fibre architecture mimicking the structures found in mantis dactyl club. Using electrospinning, we have produced uniform and highly dense microscale fibres in $3 \mathrm{D}$ helicoidal manner. The toughness of the helicoidal PCL sample is found to be nearly 5 times and 1.5 times higher than the bulk PCL and unidirectionally oriented PCL sample, respectively. Surface treatment of the helicoidal sample further enhances toughness by almost 3 times as compared to the normal 3D helicoidal sample. Mechanical testing has indeed shown some evidence of enhanced toughness in the case of the PCL helicoidal samples.

\section{Data Availability}

The (stress vs. strain) data used to support the findings of this study are included within the article.

\section{Conflicts of Interest}

The authors declare that they have no conflicts of interest.

\section{Acknowledgments}

The authors would like to gratefully acknowledge the funding from the Ministry of Education (MOE) Academic Research
Funds Tier 2 titled "Materials with Tunable Impact Resistance via Integrated Additive Manufacturing" (MOE2017-T2-2175). KA acknowledges Prof. Roland Bouffanais, Assistant Professor, Engineering Product Development (EPD) Pillar, at Singapore University of Technology and Design, for his encouragement and mentorship during her PhD study. The authors gratefully acknowledge the critical support and infrastructure provided by Singapore University of Technology and Design (SUTD) especially through the Engineering Product Design (EPD) Pillar during research work as well as during the manuscript preparation. YZ, IR, HPAA, and ASB gratefully acknowledge the critical infrastructure and capabilities in nanomechanical characterization such as provided by the Xtreme Materials Laboratory (XML). The XML Nanomechanical Characterization Laboratory capability was built through the grant funding and support provided by SUTD-MIT International Design Centre (IDC), Singapore, through the project under IDC Grant "(IDG31400102) Designing Nanomaterials Through Atomic Engineering of Interfaces." YZ, HPAA, IR, AB, and ASB also gratefully acknowledge receipt of funding and support from TEMASEK LAB@SUTD Singapore, through its SEED grant program for the project IGDS S15 01011: titled "Biomimetic, Strong yet Tough Composite through 3D Printing." KA, AB, and ASB also gratefully acknowledge receipt of funding and support from SMART (Singapore-MIT Alliance for Research and Technology) through its Ignition grant program for the project SMART ING-000067 ENG IGN: titled "Development of Novel Impact-Resistant Bio-Inspired Materials using Novel 3D Fabrication Technique." This work was supported by the SUTD-MIT International Design Centre (IDC), Singapore (IDG31400102); TEMASEK LAB@SUTD Singapore, Singapore (IGDS 515 01011); and the Singapore-MIT Alliance for Research and Technology, Singapore (SMART ING000067), also by the Ministry of Education (MOE) Academic Research Funds Tier 2 titled "Materials with Tunable Impact Resistance via Integrated Additive Manufacturing" (MOE2017-T2-2-175).

\section{References}

[1] H. J. Gao, B. H. Ji, I. L. Jager, E. Arzt, and P. Fratzl, "Materials become insensitive to flaws at nanoscale: lessons from nature," Proceedings of the National Academy of Sciences, vol. 100, no. 10, pp. 5597-5600, 2003.

[2] D. Dimitrov, K. Schreve, and N. de Beer, "Advances in three dimensional printing - state of the art and future perspectives," Rapid Prototyping Journal, vol. 12, no. 3, pp. 136-147, 2006.

[3] E. E. de Obaldia, C. Jeong, L. K. Grunenfelder, D. Kisailus, and P. Zavattieri, "Analysis of the mechanical response of biomimetic materials with highly oriented microstructures through 3D printing, mechanical testing and modeling," Journal of the Mechanical Behavior of Biomedical Materials, vol. 48, pp. 70-85, 2015.

[4] A. Sellinger, P. M. Weiss, A. Nguyen et al., "Continuous selfassembly of organic-inorganic nanocomposite coatings that mimic nacre," Nature, vol. 394, no. 6690, pp. 256-260, 1998.

[5] R. Z. Wang, Z. Suo, A. G. Evans, N. Yao, and I. A. Aksay, "Deformation mechanisms in nacre," Journal of Materials Research, vol. 16, no. 9, pp. 2485-2493, 2001. 
[6] J. C. Weaver, G. W. Milliron, A. Miserez et al., "The stomatopod dactyl club: a formidable damage-tolerant biological hammer," Science, vol. 336, no. 6086, pp. 1275-1280, 2012.

[7] S. Amini, M. Tadayon, S. Idapalapati, and A. Miserez, "The role of quasi-plasticity in the extreme contact damage tolerance of the stomatopod dactyl club," Nature Materials, vol. 14, no. 9, pp. 943-950, 2015.

[8] B. D. Wilts, H. M. Whitney, B. J. Glover, U. Steiner, and S. Vignolini, "Natural helicoidal structures: morphology, selfassembly and optical properties," Materials Today: Proceedings, vol. 1, pp. 177-185, 2014.

[9] J. Lian and J. Wang, "Microstructure and mechanical anisotropy of crab cancer magister exoskeletons," Experimental Mechanics, vol. 54, no. 2, pp. 229-239, 2014.

[10] P. Y. Chen, A. Y. M. Lin, J. McKittrick, and M. A. Meyers, "Structure and mechanical properties of crab exoskeletons," Acta Biomaterialia, vol. 4, no. 3, pp. 587-596, 2008.

[11] T. J. Zhang, Y. R. Ma, K. Chen et al., "Structure and mechanical properties of a pteropod shell consisting of interlocked helical aragonite nanofibres," Angewandte Chemie International Edition, vol. 50, no. 44, pp. 10361-10365, 2011.

[12] S. Amini, A. Masic, L. Bertinetti et al., "Textured fluorapatite bonded to calcium sulphate strengthen stomatopod raptorial appendages," Nature Communications, vol. 5, p. 12, 2014.

[13] G. X. Gu, M. Takaffoli, A. J. Hsieh, and M. J. Buehler, "Biomimetic additive manufactured polymer composites for improved impact resistance," Extreme Mechanics Letters, vol. 9, pp. 317-323, 2016.

[14] N. A. Yaraghi, N. Guarin-Zapata, L. K. Grunenfelder et al., "A sinusoidally architected helicoidal biocomposite," Advanced Materials, vol. 28, no. 32, pp. 6835-6844, 2016.

[15] A. Baji, Y. W. Mai, M. Abtahi, S. C. Wong, Y. Liu, and Q. Li, "Microstructure development in electrospun carbon nanotube reinforced polyvinylidene fluoride fibres and its influence on tensile strength and dielectric permittivity," Composites Science and Technology, vol. 88, pp. 1-8, 2013.

[16] A. Baji, Y. W. Mai, X. S. Du, and S. C. Wong, "Improved tensile strength and ferroelectric phase content of selfassembled polyvinylidene fluoride fibre yarns," Macromolecular Materials and Engineering, vol. 297, no. 3, pp. 209-213, 2012.

[17] A. Budiman, S. Han, J. Greer, N. Tamura, J. Patel, and W. Nix, "A search for evidence of strain gradient hardening in $\mathrm{Au}$ submicron pillars under uniaxial compression using synchrotron X-ray microdiffraction," Acta Materialia, vol. 56, no. 3, pp. 602-608, 2008.

[18] A. S. Budiman, G. Lee, M. J. Burek et al., "Plasticity of indium nanostructures as revealed by synchrotron X-ray microdiffraction," Materials Science and Engineering: A, vol. 538, pp. 89-97, 2012.

[19] I. Radchenko, S. Tippabhotla, N. Tamura, and A. Budiman, "Probing phase transformations and microstructural evolutions at the small scales: synchrotron X-ray microdiffraction for advanced applications in 3D IC (integrated circuits) and solar PV (photovoltaic) devices," Journal of Electronic Materials, vol. 45, no. 12, pp. 6222-6232, 2016.

[20] A. S. Budiman, S. M. Han, N. Li et al., "Plasticity in the nanoscale $\mathrm{Cu} / \mathrm{Nb}$ single-crystal multilayers as revealed by synchrotron Laue x-ray microdiffraction," Journal of Materials Research, vol. 27, no. 3, pp. 599-611, 2012.

[21] U. Chakkingal, A. B. Suriadi, and P. F. Thomson, "The development of microstructure and the influence of processing route during equal channel angular drawing of pure aluminum," Materials Science and Engineering: A, vol. 266, no. 1-2, pp. 241-249, 1999.

[22] U. Chakkingal, A. B. Suriadi, and P. Thomson, "Microstructure development during equal channel angular drawing of $\mathrm{Al}$ at room temperature," Scripta Materialia, vol. 39, no. 6, pp. 677-684, 1998.

[23] V. Handara, I. Radchenko, S. Tippabhotla et al., "Probing stress and fracture mechanism in encapsulated thin silicon solar cells by synchrotron X-ray microdiffraction," Solar Energy Materials and Solar Cells, vol. 162, pp. 30-40, 2017.

[24] D. Ginzburg, F. Pinto, O. Lervolino, and M. Meo, "Damage tolerance of bio-inspired helicoidal composites under low velocity impact," Composite Structures, vol. 161, pp. 187-203, 2017.

[25] S. K. Tippabhotla, I. Radchenko, W. Song et al., "From cells to laminate: probing and modeling residual stress evolution in thin silicon photovoltaic modules using synchrotron X-ray micro-diffraction experiments and finite element simulations," Progress in Photovoltaics: Research and Applications, vol. 25, no. 9, pp. 791-809, 2017.

[26] H. P. Anwar Ali, I. Radchenko, J. Zhou, L. Qing, and A. Budiman, "Designing novel multilayered nanocomposites for high-performance coating materials with online strain monitoring capability," Proceedings of the Institution of Mechanical Engineers, Part L: Journal of Materials: Design and Applications, 2017.

[27] Z. W. Ma, W. He, T. Yong, and S. Ramakrishna, "Grafting of gelatin on electrospun poly(caprolactone) nanofibres to improve endothelial cell spreading and proliferation and to control cell orientation," Tissue Engineering, vol. 11, no. 7-8, pp. 1149-1158, 2005.

[28] Y. Zhu, C. Gao, X. Liu, and J. Shen, "Surface modification of polycaprolactone membrane via aminolysis and biomacromolecule immobilization for promoting cytocompatibility of human endothelial cells," Biomacromolecules, vol. 3, no. 6, pp. 1312-1319, 2002.

[29] S. E. Kim, A. M. Jordan, L. T. J. Korley, and J. K. Pokorski, "Drawing in poly( $\varepsilon$-caprolactone) fibres: tuning mechanics, fibre dimensions and surface-modification density," Journal of Materials Chemistry B, vol. 5, no. 23, pp. 4499-4506, 2017.

[30] F. B. Khambatta, F. Warner, T. Russell, and R. S. Stein, "Smallangle $\mathrm{x}$-ray and light scattering studies of the morphology of blends of poly( $\epsilon$-caprolactone) with poly(vinyl chloride)," Journal of Polymer Science: Polymer Physics Edition, vol. 14, no. 8, pp. 1391-1424, 1976. 


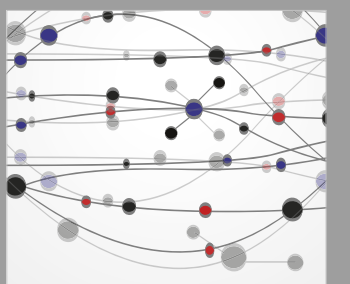

The Scientific World Journal
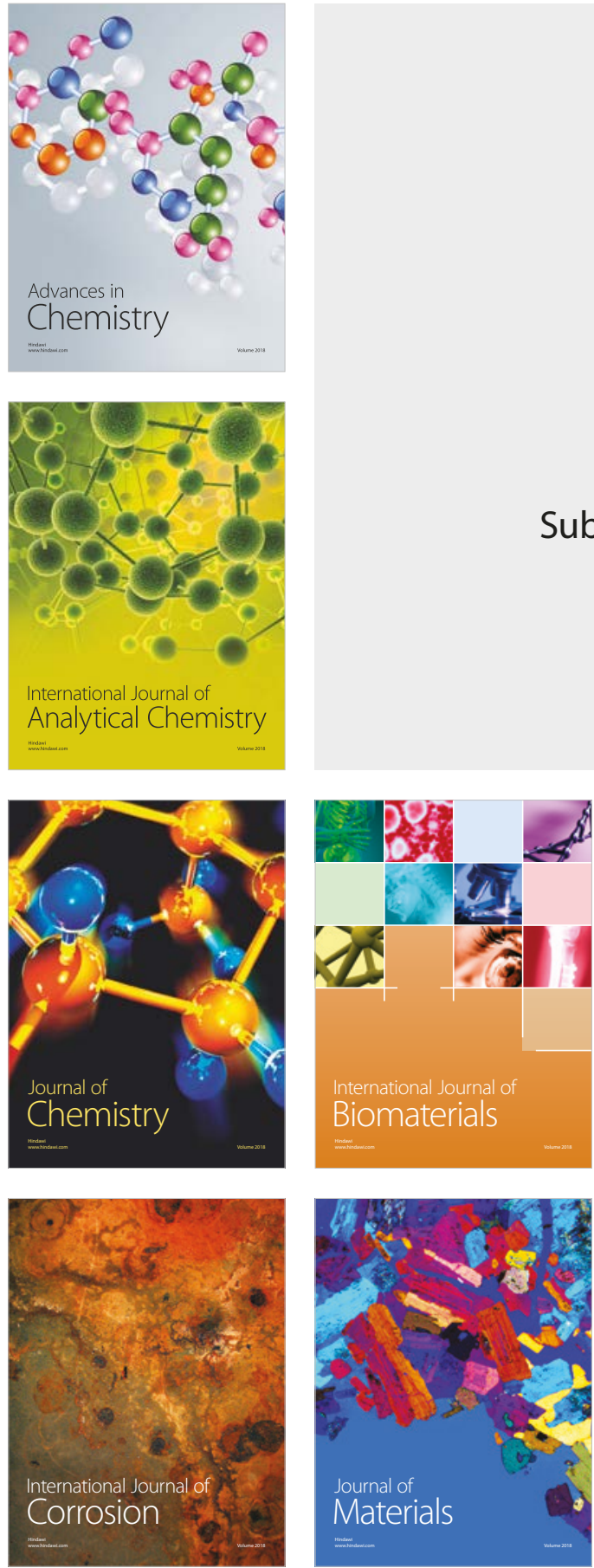



Journal of

Applied Chemistry
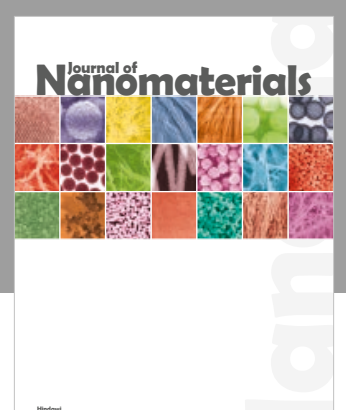



Scientifica

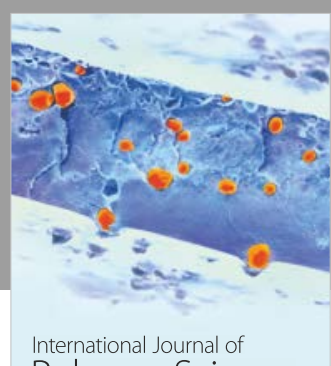

Polymer Science



Physical Chemistry
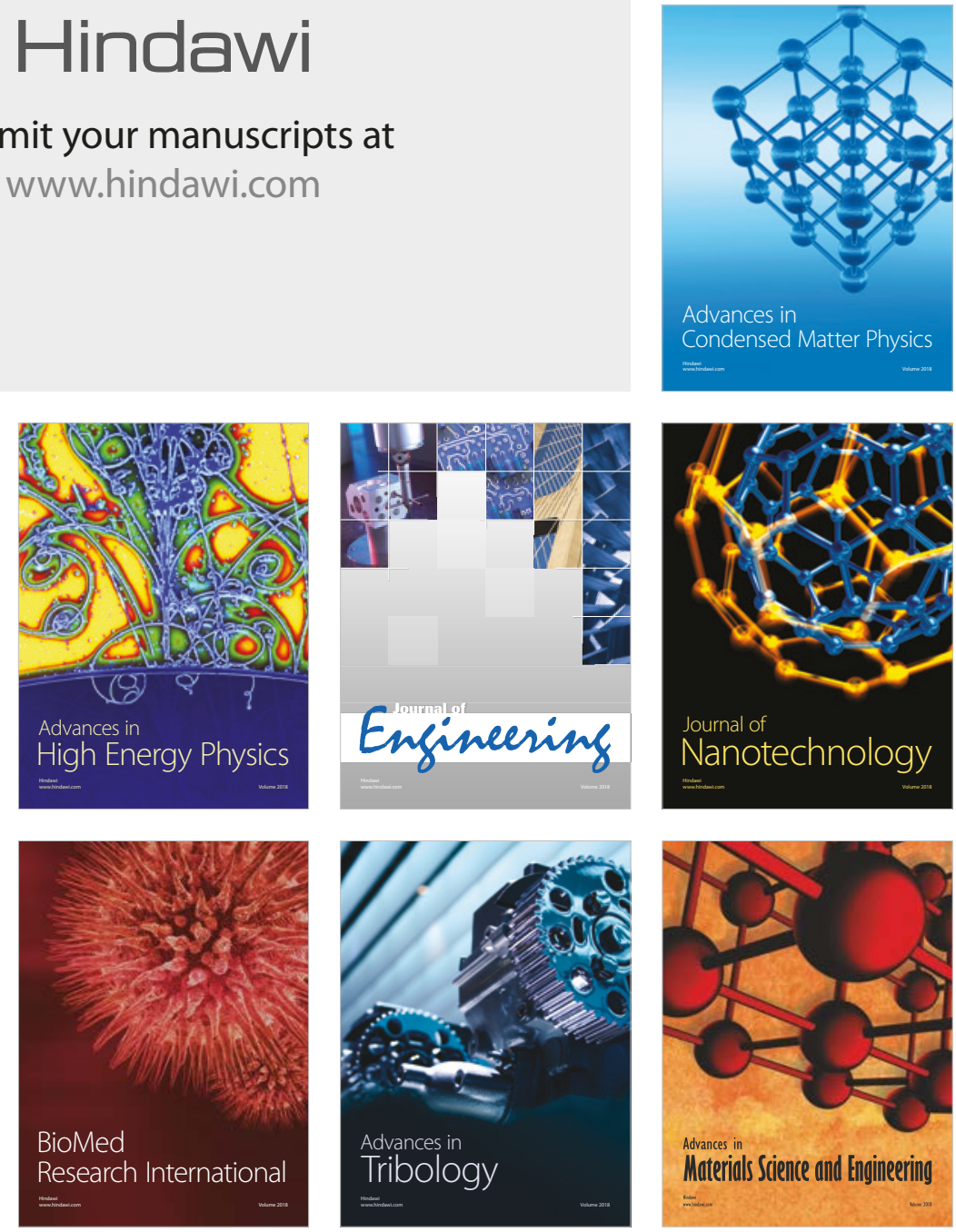